\title{
Extracorporeal Membrane Oxygenation Therapy for Critically Ill Coronavirus Disease 2019 Patients in Wuhan, China: A Retrospective Multicenter Cohort Study*
}

\author{
Jing FANG ${ }^{1-5 \dagger}$, Rui $\mathrm{LI}^{1-5 \dagger}$, Yue CHEN ${ }^{1 \dagger}$, Juan-juan QIN ${ }^{6,7 \dagger}$, Ming $\mathrm{HU}^{8 \dagger}$, Chao-lin HUANG ${ }^{9 \dagger}$, Lin $\mathrm{CHENG}^{1}, \mathrm{Yi} \mathrm{HE}^{1}$, Yi LI ${ }^{1}$, \\ Qiang ZHOU ${ }^{10}$, Dai-xing ZHOU ${ }^{11}$, Fei HUANG ${ }^{1}$, Fang $\mathrm{LEI}^{7}{ }^{12}$, Bo YANG ${ }^{13}$, Jun CHEN ${ }^{1-5 \#}$, Hong-ping DENG ${ }^{14}$, \\ Yu-feng YUAN ${ }^{15 \#}$, Jia-hong XIA ${ }^{16 \#}$, Song WAN ${ }^{17 \#}$, Hong-liang LI $^{6,7 \#}$, Xiang WEI ${ }^{1-5 \#}$ \\ ${ }^{I}$ Division of Cardiothoracic and Vascular Surgery, Tongji Hospital, Tongji Medical College, Huazhong University of Science \\ and Technology, Wuhan 430030, China \\ ${ }^{2}$ Key Laboratory of Organ Transplantation, Ministry of Education, Wuhan 430030, China \\ ${ }^{3}$ NHC Key Laboratory of Organ Transplantation, Ministry of Health, Wuhan 430030, China \\ ${ }^{4}$ Key Laboratory of Organ Transplantation, Chinese Academy of Medical Sciences, Wuhan 430030, China \\ ${ }^{5}$ National Public Health Event Medical Center, National Health Commission of the People's Republic of China, Wuhan 430030 , \\ China \\ ${ }^{6}$ Department of Cardiology, Renmin Hospital of Wuhan University, Wuhan 430060, China \\ ${ }^{7}$ Basic Medical School, Wuhan University, Wuhan 430071, China \\ ${ }^{8}$ Department of Critical Care Medicine, Wuhan Pulmonary Hospital, Wuhan 430030, China \\ ${ }^{9}$ Wuhan Jinyintan Hospital, Wuhan 430023, China \\ ${ }^{10}$ Division of Cardiology, Department of Internal Medicine, Tongji Hospital, Tongji Medical College, Huazhong University of \\ Science and Technology, Wuhan 430030, China \\ ${ }^{11}$ Department of Intensive Critical Medicine, Tongji Hospital, Tongji Medical College, Huazhong University of Science and \\ Technology, Wuhan 430030, China \\ ${ }^{12}$ Institute of Model Animal of Wuhan University, Wuhan 430071, China \\ ${ }^{13}$ Department of Cardiac Surgery, University of Michigan, Ann Arbor, MI 48109, USA \\ ${ }^{14}$ Department of Vascular Surgery, Renmin Hospital of Wuhan University, Wuhan 430060, China \\ ${ }^{15}$ Department of Hepatobiliary and Pancreatic Surgery, Zhongnan Hospital of Wuhan University, Wuhan 430071, China \\ ${ }^{16}$ Department of Cardiovascular Surgery, Union Hospital, Tongji Medical College, Huazhong University of Science and \\ Technology, Wuhan 430022, China \\ ${ }^{17}$ Division of Cardiothoracic Surgery, Department of Surgery, the Chinese University of Hong Kong, Prince of Wales Hospital, \\ Hong Kong, 999077 China
}

(C) Huazhong University of Science and Technology 2021

Summary: Currently, little in-depth evidence is known about the application of extracorporeal membrane oxygenation (ECMO) therapy in coronavirus disease 2019 (COVID-19) patients. This retrospective multicenter cohort study included patients with COVID-19 at 7 designated hospitals in Wuhan, China. The patients were followed up until June 30, 2020. Univariate

Jing FANG, E-mail: jingfang@hust.edu.cn; Rui LI, E-mail: ruilee_tj@126.com; Yue CHEN, E-mail: chen_yue@hust. edu.cn; Juan-juan QIN, E-mail: qinjuanjuan@whu.edu.cn; Ming HU, E-mail: huming74@163.com; Chao-lin HUANG, E-mail:88071718@qq.com

${ }^{\dagger}$ The authors contributed equally to this work.

\#Corresponding authors, Jun CHEN, E-mail: chjunmage@, hotmail.com; Hong-ping DENG, E-mail: hpdeng@whu.edu. cn; Yu-feng YUAN, E-mail: yuanyf1971@whu.edu.cn; Jiahong XIA, E-mail: jiahong.xia@hust.edu.cn; Song WAN, E-mail: swan@surgery.cuhk.edu.hk; Hong-liang LI, E-mail: lihl@whu.edu.cn; Xiang WEI, E-mail: xiangwei@tjh.tjmu. edu.cn

*This work was supported by grants from Emergent Key Projects for COVID-19 (No. 2020kfyXGYJ091), the National Natural Science Foundation of China (Nos. 81800256, 81873458, 81670050), and National Key Research and Development Program of China (No. 2019YFC0121600). and multivariate logistic regression analyses were performed to identify the risk factors associated with unsuccessful ECMO weaning. Propensity score matching was used to match patients who received veno-venous ECMO with those who received invasive mechanical ventilation (IMV)-only therapy. Of 88 patients receiving ECMO therapy, 27 and 61 patients were and were not successfully weaned from ECMO, respectively. Additionally, 15, 15, and 65 patients were further weaned from IMV, discharged from hospital, or died during hospitalization, respectively. In the multivariate logistic regression analysis, a lymphocyte count $\leq 0.5 \times 10^{9}$ / $\mathrm{L}$ and D-dimer concentration $>4 \times$ the upper limit of normal level at ICU admission, a peak $\mathrm{PaCO}_{2}>60 \mathrm{mmHg}$ at $24 \mathrm{~h}$ before ECMO initiation, and no tracheotomy performed during the ICU stay were independently associated with lower odds of ECMO weaning. In the propensity scorematched analysis, a mixed-effect Cox model detected a lower hazard ratio for 120-day all-cause mortality after ICU 
admission during hospitalization in the ECMO group. The presence of lymphocytopenia, higher D-dimer concentrations at ICU admission and hypercapnia before ECMO initiation could help to identify patients with a poor prognosis. Tracheotomy could facilitate weaning from ECMO. ECMO relative to IMV-only therapy was associated with improved outcomes in critically ill COVID-19 patients.

Key words: coronavirus disease 2019; extracorporeal membrane oxygenation; mechanical ventilation; acute respiratory distress syndrome; viral pneumonia

On April 26, 2020, the number of hospitalized coronavirus disease 2019 (COVID-19) cases decreased to zero in Wuhan, the Chinese city most severely affected by the COVID-19 pandemic. However, the global pandemic situation remains critical, as the number of new severe acute respiratory syndrome coronavirus 2 (SARS-CoV-2) infection cases continues to increase without signs of alleviation. Recent studies have revealed that up to $12.2 \%$ of hospitalized COVID-19 patients in the USA were refractory to oxygen therapy and required advanced forms of respiratory support, such as invasive mechanical ventilation (IMV) ${ }^{[1]}$. COVID-19-induced acute respiratory distress syndrome (ARDS) is characterized by a decrease in lung compliance, which necessitates a high IMV driving pressure to maintain oxygenation and may consequently cause ventilator-induced lung injury. This led to disappointing outcomes of IMV in critically ill COVID-19 patients and mortality rates as high as $88.1 \%{ }^{[1]}$. Extracorporeal membrane oxygenation (ECMO) therapy can support not only ventilation but also gas exchange. Such a complete lung replacement therapy would guarantee both oxygen intake and $\mathrm{CO}_{2}$ removal. This aspect is particularly important because a deficiency in gas exchange has been identified as the dominant cause of hypoxemia in COVID-19 patients who develop ARDS. Moreover, the use of ECMO may protect the lungs, in contrast to IMV, and this could better enable viral depletion and lung recovery.

ECMO has been widely used as a life-saving therapy in critically ill patients with viral pneumonia since the demonstration of its superiority over IMV during the 2009 H1N1 influenza pandemic ${ }^{[2,}$ ${ }^{3]}$. Although sporadic studies have reported the use of ECMO in COVID-19 patients, the results were generally disappointing ${ }^{[4,5]}$. This study aimed to systematically and comprehensively elaborate the efficacy, therapeutic considerations, and outcomes of ECMO application in COVID-19 patients in Wuhan, the largest cohort in China. We sought to identify the risk factors that accounted for unsuccessful ECMO weaning, so as to facilitate decision making regarding eligibility for ECMO implementation. Using propensity score matching, we matched ECMO and IMV patients to compare the effectiveness and outcomes of the two approaches, using 120-day in-hospital mortality after ICU admission as the primary end point. We hypothesized that in critically ill COVID-19 patients, ECMO use would be associated with an improved outcome when compared to IMV-only therapy.

\section{PATIENTS AND METHODS}

\subsection{Study Design and Patient Enrollment}

This retrospective, multicenter study included patients who had been diagnosed with COVID-19 according to the Guidelines on Diagnosis and Treatment for New Coronavirus Pneumonia (5th edition), published by the National Health Commission of the People's Republic of China. Seven designated local hospitals in Wuhan that were temporarily transformed for treating COVID-19 and providing ECMO therapy for more than 5 patients with COVID-19 were included in the present study: Tongji Hospital and Union Hospital of Huazhong University of Science and Technology, Zhongnan Hospital and Renmin Hospital of Wuhan University, Wuhan Jinyintan Hospital, Wuhan Pulmonary Hospital, and Leishenshan Hospital. In total, 94 patients received ECMO therapy and 536 received IMV-only therapy. The institutional ethical committee of each participating hospital approved the study protocol and waived the requirement for informed patient consent. The patients' clinical outcomes during hospitalization were monitored until June 30, 2020. For patients who were transferred between hospitals, the courses were merged and considered as a single course. Patients without a definite outcome (e.g., weaned from ECMO, discharged, or deceased) because of incomplete medical records were excluded.

\subsection{Initiation, Management, and Weaning of ECMO}

The indications and contradictions of ECMO therapy were determined by the local ECMO team in accordance with the Guidelines on the Diagnosis and Treatment for New Coronavirus Pneumonia of Severely and Critically Ill Patients (2nd edition) released by the National Health Commission of the People's Republic of China. The multidisciplinary ECMO team comprised intensive care specialists, cardiac surgeons, perfusionists, and nursing personnels. ECMO was considered when protective ventilation and prone-position ventilation were ineffective and one or more of the following criteria were met, despite the application of an optimal ventilatory strategy [fraction of inspired oxygen $\left(\mathrm{FiO}_{2}\right) \geq 0.8$, tidal volume of $6 \mathrm{~mL} /$ 
$\mathrm{kg}$ of predicted body weight, positive end-expiratory pressure (PEEP) $\geq 10 \mathrm{cmH}_{2} \mathrm{O}$ ]: (1) partial pressure of oxygen $\left(\mathrm{PaO}_{2}\right)$ : $\mathrm{FiO}_{2}<50 \mathrm{mmHg}>3 \mathrm{~h} ;(2) \mathrm{PaO}_{2}: \mathrm{FiO}_{2}$ $<80 \mathrm{mmHg}>6 \mathrm{~h}$; (3) $\mathrm{PaO}_{2}: \mathrm{FiO}_{2}<100 \mathrm{mmHg}$ at a $\mathrm{FiO}_{2}=1.0$; (4) arterial $\mathrm{pH}<7.25$ and $\mathrm{PaCO}_{2}>60 \mathrm{mmHg}$ $>6 \mathrm{~h}$ with a respiratory rate $>35$ breaths $/ \mathrm{min}$; (5) arterial $\mathrm{pH}<7.20$ with a respiratory rate $>35$ breaths $/ \mathrm{min}$ and plateau pressure $>30 \mathrm{cmH}_{2} \mathrm{O}$; (6) cardiogenic shock or cardiac arrest. Relative contradictions included (1) a combination of irreversible diseases; (2) contradictions to anticoagulation; (3) mechanical ventilation $>7$ days under high ventilatory settings $\left(\mathrm{FiO}_{2}>0.9\right.$ and plateau pressure $>30 \quad \mathrm{cmH}_{2} \mathrm{O}$ ); (4) immunocompromised status; or (5) lack of vascular access for ECMO because of vascular deformity. Veno-venous (V-V) ECMO was preferred for patients with normal cardiac function. Veno-arterial (V-A) ECMO was applied when cardiogenic shock was diagnosed. ECMO management and weaning were performed at the multidisciplinary team's discretion when the lung function was improved and lung abnormalities were resolved. The details and weaning course were as previously described ${ }^{[6]}$.

\subsection{Data Collection}

We obtained the electronic medical records from hospitals and extracted data concerning the patients' demographic information, comorbidities, medical histories, laboratory and imageological findings, inpatient medications, medical orders, treatments, daily progress notes, nursing records, and final outcomes (i.e., ICU discharge, hospital discharge, and mortality). The patients' names and IDs were anonymized to protect privacy before data extraction. All data were reviewed by experienced clinicians, entered into a computerized database, and double-checked. If essential data were missing in the electronic records, we contacted the corresponding physicians-in-charge directly.

\subsection{Definitions}

The onset of COVID-19 was defined as the first date when the symptoms were noticed. Patients who were successfully weaned from ECMO and remained alive for at least $48 \mathrm{~h}$ were classified as the weaned group. Patients who died despite receiving ECMO therapy or remained on ECMO until June 30, 2020, were classified as the non-weaned group. Acute kidney injury was identified as an increase of $\geq 26.5 \mu \mathrm{mol} / \mathrm{L}$ in the serum creatinine concentration within $48 \mathrm{~h}^{[7]}$. Acute cardiac injury was identified as a serum cardiac troponin I (cTnI) concentration above the upper limit of normal (ULN). Acute hepatic injury was defined as an increase in the serum aspartate aminotransferase or alanine aminotransferase (ALT) concentration to more than three times the ULN ${ }^{[8]}$. Disseminated intravascular coagulation (DIC) was defined according to the criteria set by the International Society on Thrombosis and Hemostasis ${ }^{[9]}$. The following criteria for discharge were applied as recommended by the guidelines: (1) throat swab or sputum specimens collected at an interval of at least $24 \mathrm{~h}$ yielded negative SARS-CoV-2 test results; (2) body temperature was normal for 3 consecutive days; (3) symptoms of COVID-19 were resolved; and (4) chest computed tomography manifestations of COVID-19 were significantly improved. Nosocomial infection was diagnosed in patients who exhibited signs of bacteremia or pneumonia and a positive pathogen culture from lower respiratory tract specimens (e.g., qualified sputum, endotracheal aspirate, or bronchoalveolar lavage fluid) or blood samples at $48 \mathrm{~h}$ after hospital admission.

\subsection{Propensity Score-matched Analysis}

Propensity score-matched cohorts of patients who received ECMO therapy or IMV-only therapy were created based on variables expected to be confounders associated with the outcomes of ECMO or IMVonly treatment. These variables included age, sex, comorbidities (hypertension, diabetes), and laboratory examination findings at ICU admission [lymphocyte count, C-reactive protein, creatintine, total bilirubin, ALT, lactate dehydrogenase (LDH), N-terminal prohormone brain natriuretic peptide (NT-proBNP) or BNP, and D-dimer]. Only patients who received $\mathrm{V}-\mathrm{V}$ ECMO treatment were included in the ECMO group for this cohort analysis (fig. 1). We used nonparametric missing value imputation based on the missForest procedure on the $\mathrm{R}$ platform to account for missing laboratory variable data ${ }^{[10]}$. A random forest model that included the remaining variables in the data set was performed to predict the missing values for those variables. Internally cross-validated errors were also estimated. Patients who were died within $24 \mathrm{~h}$ after ECMO or IMV initiation were excluded. ECMO and IMV-only patients were paired at a 1:1 ratio according to the propensity scores, using exact matching with a caliper size of 0.05 . The balance between covariates was evaluated by estimating the standardized differences before and after matching. Only standardized differences with absolute values of $<0.1$ were considered to indicate qualified balancing. A mixed-effect Cox model was used to compare the two treatment groups in terms of the primary endpoint, which was 120-day all-cause mortality after ICU admission during hospitalization.

\subsection{Statistical Analysis}

Continuous variables are presented as medians and interquartile ranges (IQRs), and categorical variables are expressed as number $(n)$ and corresponding percentages. Continuous variables and ordered categorical variables were analyzed using the MannWhitney $U$ test, whereas proportions of unordered categorical variables were compared using the $\chi^{2}$ test or Fisher's exact test when the sample sizes were small. Univariate logistic regression models were initially applied to analyze the association between each 
single variable and the final weaning of ECMO, and indices with $P$ values $<0.1$ were included in a further multivariate logistic regression. The odds ratios (ORs) and $95 \%$ confidence intervals (CIs) were reported.

The risk of reaching the primary endpoint and the corresponding hazard ratio (HR) were calculated using the mixed-effect Cox model and compared between the ECMO and IMV-only groups. We adjusted multiple variables considered relevant to the endpoint, including age, sex, comorbidities (hypertension, chronic lung disease), laboratory examination findings at ICU admission (platelet, total bilirubin, LDH, NTproBNP or BNP, creatinine, D-dimer), and treatments during the ICU stay (prone position ventilation, convalescent plasma). We modeled the site as a random effect in the mixed-effect Cox model. The proportional hazard assumptions were verified using correlation testing based on Schoenfeld residuals. The cumulative death rates were compared using the Kaplan-Meier method. A two-sided $P$ value of $<0.05$ was considered statistically significant. The data were analyzed using the $\mathrm{R}$ platform version 3.6.3 with the "tableone" and "stats" packages (R Foundation for Statistical Computing, Vienna, Austria), STATA software version 15.0 (Stata Corp., College Station, USA) and SPSS Statistics version 23.0 (IBM, Armonk, USA).

\section{RESULTS}

\subsection{Clinical Characteristics of COVID-19 Patients Who Received ECMO Therapy in Wuhan \\ Ninety-four critically ill COVID-19 patients}

received ECMO therapy at 7 designated hospitals in Wuhan before June 30, 2020. Six patients without final results due to incomplete medical records were excluded. Of the remaining 88 patients, 27 were successfully weaned (weaned group) and 61 were unsuccessfully weaned (non-weaned group) from ECMO (fig. 1). Figure 2 presents the number of patients concurrently treated with ECMO in Wuhan between January 1 and June 30. ECMO was first implemented on January 2, and concurrent ECMO cases peaked on March 7 ( $n=36$; fig. 2). The first patient in the weaned group received ECMO support on January 28 (fig. $2)$. Patients in the non-weaned group had a median (IQR) age of $62.00(52.00-68.00)$ years and were significantly older than those in the weaned group [median: 50.00 (42.00-64.00) years; table 1)]. The male sex was predominant in both groups (table 1). The median (IQR) duration from symptom onset to first admission was $12.00(7.00-20.25)$ days (table 1). Approximately half of the patients had comorbidities, of which hypertension and diabetes mellitus were the most frequent (table 1).

The majority of patients presented with lymphocytopenia at the time of ICU admission, which was more severe in the non-weaned group (table 1). Similarly, small proportions of patients in both groups demonstrated thrombocytopenia and elevated total bilirubin, ALT, and creatinine concentrations (table 1). Similarly, large proportions of patients in both groups exhibited substantially elevated serum C-reactive protein, LDH, D-dimer, and IL-6 concentrations (table 1). Nearly half of the patients exhibited elevated NT-

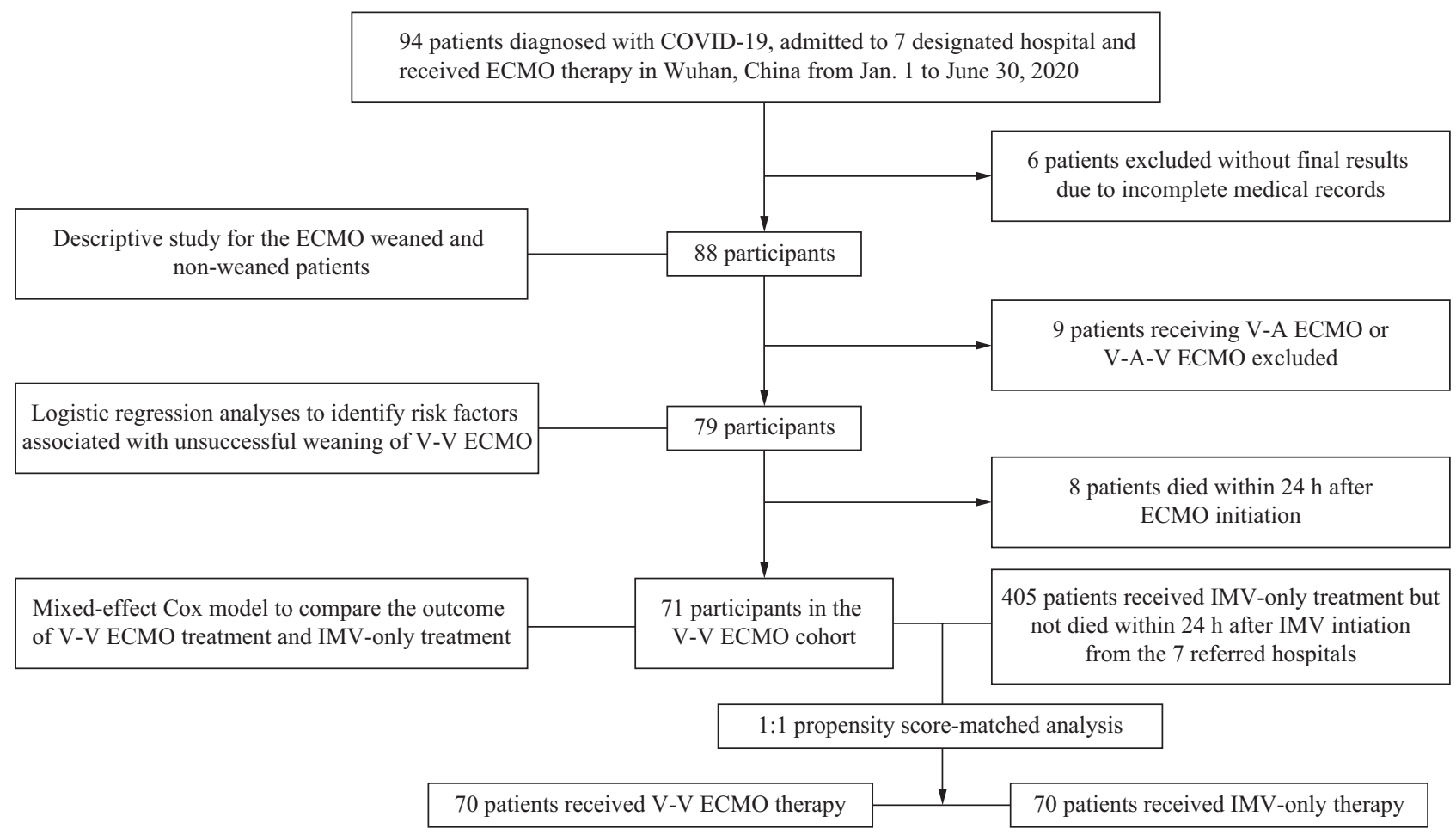

Fig. 1 Flow diagram of patient enrollment and propensity score matching in this study 
Table 1 Demographic and clinical characteristics of patients with COVID-19

\begin{tabular}{|c|c|c|c|c|}
\hline Parameters & All patients $(n=88)$ & Weaned $(n=27)$ & Non-weaned $(n=61)$ & $P$ value \\
\hline \multicolumn{5}{|l|}{ Clinical characteristics on hospital admission } \\
\hline Age, years & $58.50(47.00-66.50)$ & $50.00(42.00-64.00)$ & $62.00(52.00-68.00)$ & 0.038 \\
\hline $18-40$ & $8 / 88(9.1 \%)$ & $4 / 27(14.8 \%)$ & $4 / 61(6.6 \%)$ & 0.055 \\
\hline $41-60$ & $38 / 88(43.2 \%)$ & $14 / 27(51.9 \%)$ & $24 / 61(39.3 \%)$ & \\
\hline $61-70$ & $31 / 88(35.2 \%)$ & $7 / 27(25.9 \%)$ & $24 / 61(39.3 \%)$ & \\
\hline$>70$ & $11 / 88(12.5 \%)$ & $2 / 27(7.4 \%)$ & $9 / 61(14.8 \%)$ & \\
\hline Female & $32 / 88(36.4 \%)$ & $8 / 27(29.6 \%)$ & $24 / 61(39.3 \%)$ & 0.382 \\
\hline Symptom onset to first admission, days & $12.00(7.00-20.25)$ & $14.00(8.50-20.00)$ & $11.00(7.00-21.00)$ & 0.710 \\
\hline Any comorbidity & $45 / 88(51.1 \%)$ & $15 / 27(55.6 \%)$ & $30 / 61(49.2 \%)$ & 0.581 \\
\hline \multicolumn{5}{|l|}{ Major comorbidities } \\
\hline Hypertension & $35 / 88(39.8 \%)$ & $11 / 27(40.7 \%)$ & $24 / 61(39.3 \%)$ & 0.902 \\
\hline Diabetes Mellitus & $18 / 88(20.5 \%)$ & $7 / 27(25.9 \%)$ & $11 / 61(18.0 \%)$ & 0.397 \\
\hline Chronic lung disease & $1 / 88(1.1 \%)$ & $0 / 27(0.0 \%)$ & $1 / 61(1.6 \%)$ & 1.000 \\
\hline $\begin{array}{l}\text { Cardiovascular disease (excluding } \\
\text { hypertension) }\end{array}$ & $8 / 88(9.1 \%)$ & $3 / 27(11.1 \%)$ & $5 / 61(8.2 \%)$ & 0.971 \\
\hline Neurological disease & $3 / 88(3.4 \%)$ & $3 / 27(11.1 \%)$ & $0 / 61(0.0 \%)$ & 0.027 \\
\hline Chronic liver disease & $4 / 88(4.5 \%)$ & $0 / 27(0.0 \%)$ & $4 / 61(6.6 \%)$ & 0.308 \\
\hline Chronic renal disease & $0 / 88(0.0 \%)$ & $0 / 27(0.0 \%)$ & $0 / 61(0.0 \%)$ & \\
\hline Malignancy & $1 / 88(1.1 \%)$ & $0 / 27(0.0 \%)$ & $1 / 61(1.6 \%)$ & 1.000 \\
\hline \multicolumn{5}{|l|}{ Laboratory examinations on ICU admission } \\
\hline Leukocyte count, $\times 10^{9} / \mathrm{L}$ & $12.68(8.10-17.19)$ & $12.72(8.19-19.06)$ & $12.63(8.11-16.40)$ & 0.790 \\
\hline$>10 \times 10^{9} / \mathrm{L}$ & $57 / 88(64.8 \%)$ & $16 / 27(59.3 \%)$ & $41 / 61(67.2 \%)$ & 0.471 \\
\hline Lymphocyte count, $\times 10^{9} / \mathrm{L}$ & $0.65(0.39-0.86)$ & $0.75(0.50-0.96)$ & $0.54(0.32-0.84)$ & 0.065 \\
\hline$\leq 0.5 \times 10^{9} / \mathrm{L}$ & $36 / 88(40.9 \%)$ & $7 / 27(25.9 \%)$ & $29 / 61(47.5 \%)$ & 0.057 \\
\hline Platelet, $\times 10^{9} / \mathrm{L}$ & $164.50(104.75-225.75)$ & $165.00(142.00-212.50)$ & $164.00(100.00-234.00)$ & 0.825 \\
\hline$<\mathrm{LLN}$ & $27 / 88(30.7 \%)$ & $5 / 27(18.5 \%)$ & $22 / 61(36.1 \%)$ & 0.100 \\
\hline C-reactive protein, $\mathrm{mg} / \mathrm{L}$ & $95.40(56.80-160.00)$ & $86.40(29.43-138.10)$ & $96.70(64.50-160.00)$ & 0.095 \\
\hline$>100$ & $39 / 85(45.9 \%)$ & $12 / 26(46.2 \%)$ & $27 / 59(45.8 \%)$ & 0.973 \\
\hline Total bilirubin, $\mu \mathrm{mol} / \mathrm{L}$ & $13.90(9.35-21.58)$ & $11.60(9.19-17.20)$ & $14.60(10.21-25.30)$ & 0.080 \\
\hline$>\mathrm{ULN}$ & $21 / 88(23.9 \%)$ & $3 / 27(11.1 \%)$ & $18 / 61(29.5 \%)$ & 0.062 \\
\hline ALT, U/L & $37.00(21.00-61.25)$ & $39.00(25.00-80.50)$ & $36.00(21.00-54.00)$ & 0.326 \\
\hline$>\mathrm{ULN}$ & $33 / 88(37.5 \%)$ & $12 / 27(44.4 \%)$ & $21 / 61(34.4 \%)$ & 0.371 \\
\hline $\mathrm{LDH}, \mathrm{U} / \mathrm{L}$ & $547.00(432.00-700.00)$ & $531.00(436.00-647.75)$ & $571.00(432.00-736.00)$ & 0.562 \\
\hline$>1.5 \times \mathrm{ULN}$ & $69 / 80(86.25 \%)$ & $21 / 24(87.5 \%)$ & $48 / 56(85.7 \%)$ & 1.000 \\
\hline NT-proBNP, pg/mL & $225.20(73.40-968.00)$ & $195.00(84.60-1218.50)$ & $227.10(73.10-869.30)$ & 0.727 \\
\hline$>$ ULN & $40 / 87(46.0 \%)$ & $14 / 27(51.9 \%)$ & $26 / 60(43.3 \%)$ & 0.461 \\
\hline cTnI, pg/mL & $33.00(12.90-239.40)$ & $30.00(12.20-225.30)$ & $37.00(16.70-235.75)$ & 0.696 \\
\hline$>\mathrm{ULN}$ & $40 / 85(47.1 \%)$ & $11 / 26(42.3 \%)$ & $29 / 59(49.2 \%)$ & 0.560 \\
\hline Creatinine, $\mu \mathrm{mol} / \mathrm{L}$ & $66.20(51.25-97.40)$ & $65.00(46.30-85.30)$ & $69.00(54.60-101.00)$ & 0.260 \\
\hline$>\mathrm{ULN}$ & $24 / 88(27.3 \%)$ & $5 / 27(18.5 \%)$ & $19 / 61(31.1 \%)$ & 0.220 \\
\hline D-dimer, mg/L & $4.51(1.63-16.87)$ & $2.96(1.31-11.32)$ & $7.28(2.28-19.04)$ & 0.152 \\
\hline$>4 \times \mathrm{ULN}$ & $60 / 87(69.0 \%)$ & $14 / 27(51.9 \%)$ & $46 / 60(76.7 \%)$ & 0.021 \\
\hline Serum IL-6, pg/mL & $44.30(12.51-196.20)$ & $74.86(15.88-199.20)$ & $40.26(11.80-188.00)$ & 0.339 \\
\hline$>10 \times \mathrm{ULN}$ & $31 / 70(44.3 \%)$ & $11 / 23(47.8 \%)$ & $20 / 47(42.6 \%)$ & 0.677 \\
\hline \multicolumn{5}{|l|}{ Scores on ICU admission } \\
\hline Murray score & $3.00(2.70-3.30)$ & $3.00(2.67-3.15)$ & $3.00(2.70-3.33)$ & 0.146 \\
\hline Missing data & $0 / 88(0.0 \%)$ & $0 / 27(0.0 \%)$ & $0 / 61(0.0 \%)$ & \\
\hline SOFA score & $8.00(6.00-10.00)$ & $7.50(6.00-9.00)$ & $8.00(7.00-11.00)$ & 0.207 \\
\hline Missing data & $11 / 88(12.5 \%)$ & $3 / 27(11.1 \%)$ & $8 / 61(13.1 \%)$ & \\
\hline \multicolumn{5}{|l|}{ 24-h before ECMO initiation } \\
\hline Highest respiratory rate, breaths/min & $24.00(20.00-28.00)$ & $22.00(18.00-24.00)$ & $25.00(22.00-28.00)$ & 0.008 \\
\hline Highest PEEP, $\mathrm{cmH}_{2} \mathrm{O}$ & $10.00(8.00-11.00)$ & $10.00(7.50-10.00)$ & $10.00(8.00-12.00)$ & 0.123 \\
\hline Lowest $\mathrm{PaO}_{2} / \mathrm{FiO}_{2}, \mathrm{mmHg}$ & $88.75(65.79-128.27)$ & $128.85(81.50-168.73)$ & $81.41(61.50-104.81)$ & 0.015 \\
\hline$>200$ & $3 / 60(5.0 \%)$ & $2 / 16(12.5 \%)$ & $1 / 44(2.3 \%)$ & 0.067 \\
\hline $80-200$ & $32 / 60(53.3 \%)$ & $10 / 16(62.5 \%)$ & $22 / 44(50.0 \%)$ & \\
\hline$<80$ & $25 / 60(41.7 \%)$ & $4 / 16(25.0 \%)$ & $21 / 44(47.7 \%)$ & \\
\hline
\end{tabular}


(Continued from the last page)

\begin{tabular}{lcccc}
\hline Parameters & All patients $(n=88)$ & Weaned $(n=27)$ & Non-weaned $(n=61)$ & $P$ value \\
\hline Highest $\mathrm{PaCO}_{2}, \mathrm{mmHg}$ & $58.65(42.85-71.83)$ & $47.00(33.80-54.20)$ & $64.00(45.00-74.30)$ & 0.003 \\
$\quad>60$ & $31 / 66(47.0 \%)$ & $2 / 17(11.8 \%)$ & $29 / 49(59.2 \%)$ & 0.001 \\
$35-60$ & $25 / 66(37.9 \%)$ & $10 / 17(58.8 \%)$ & $15 / 49(30.6 \%)$ & $5 / 49(10.2 \%)$ \\
$\quad<35$ & $10 / 66(15.2 \%)$ & $5 / 17(29.4 \%)$ & $7.36(7.26-7.42)$ & 0.045 \\
Lowest arterial pH & $7.38(7.27-7.43)$ & $7.41(7.36-7.47)$ & $8 / 48(16.7 \%)$ & 0.283 \\
$\quad>7.45$ & $13 / 65(20.0 \%)$ & $5 / 17(29.4 \%)$ & $19 / 48(39.6 \%)$ & \\
$7.35-7.45$ & $27 / 65(41.5 \%)$ & $8 / 17(47.1 \%)$ & $21 / 48(43.8 \%)$ & \\
$\quad<7.35$ & $25 / 65(38.5 \%)$ & $4 / 17(23.5 \%)$ & $2.10(1.50-3.60)$ & 0.560 \\
Highest arterial lactic acid, mmol/L & $2.10(1.50-3.00)$ & $2.05(1.31-2.80)$ & $20 / 35(57.1 \%)$ & 0.851 \\
$\quad>$ ULN & $29 / 50(58.0 \%)$ & $9 / 15(60.0 \%)$ & & \\
Treatments during ICU stay & & & & \\
Requirement of inotropes or vasopressors & $26 / 87(29.9 \%)$ & $8 / 26(30.8 \%)$ & $18 / 61(29.5 \%)$ & \\
$\quad$ before intubation & & & & \\
Anti-viral treatment & $52 / 88(59.1 \%)$ & $17 / 27(63.0 \%)$ & $35 / 61(57.4 \%)$ & 0.623 \\
Anti-microbial treatment & $87 / 88(98.9 \%)$ & $27 / 27(100.0 \%)$ & $60 / 61(98.4 \%)$ & 1.000 \\
Anti-fungi treatment & $58 / 88(65.9 \%)$ & $16 / 27(59.3 \%)$ & $42 / 61(68.9 \%)$ & 0.381 \\
Convalescent plasma & $19 / 88(21.6 \%)$ & $12 / 27(44.4 \%)$ & $7 / 61(11.5 \%)$ & 0.001 \\
Corticosteroids & $57 / 88(64.8 \%)$ & $20 / 27(74.1 \%)$ & $37 / 61(60.7 \%)$ & 0.224 \\
IVIG & $50 / 88(56.8 \%)$ & $16 / 27(59.3 \%)$ & $34 / 61(55.7 \%)$ & 0.758 \\
\hline
\end{tabular}

Data are median (IQR) or $n(\%) . P$ values were calculated by Mann-Whitney $U$ test, $\chi^{2}$ test, or Fisher's exact test, as appropriate. Upper limit of normal (ULN) and lower limit of normal (LLN) were defined according to the normal ranges of tests in each hospital. ALT: alanine transaminase; LDH: lactate dehydrogenase: NT-proBNP: N-terminal pro-brain natriuretic peptide; cTnI: cardiac troponin I; IL-6: interleukin-6; SOFA: sequential organ failure assessment; PEEP: positive end expiratory pressure; $\mathrm{PaO}_{2} / \mathrm{FiO}_{2}$ : partial pressure of arterial oxygen/fraction of inspiration oxygen; $\mathrm{PaCO}_{2}$ : partial pressure of arterial carbon dioxide; IVIG: intravenous immunoglobulin

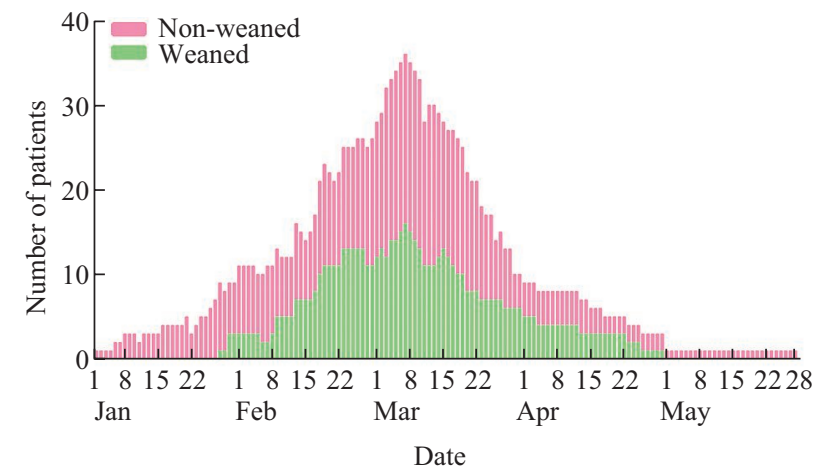

Fig. 2 Histogram of the number of patients concurrently treated with ECMO in Wuhan, China, from January 1 to June 30, 2020. Green and red histograms indicate the patients finally successfully weaned (weaned group) and failed to wean (non-weaned) from ECMO, respectively.

proBNP and cTnI concentrations indicative of an abnormal cardiac status (table 1). The median Murray score, which is used to assess the severity of ARDS, was 3.0 in both groups (table 1). The median sequential organ failure assessment (SOFA) score, which is used to evaluate the functions of extra-pulmonary organs, was 8.00 (IQR: 6.00-10.00).

The non-weaned group had a significantly higher peak respiratory rate and peak $\mathrm{PaCO}_{2}$ at $24 \mathrm{~h}$ before ECMO initiation and a significantly lower minimum $\mathrm{PaO}_{2} / \mathrm{FiO}_{2}$ and arterial $\mathrm{pH}$ than in the weaned group (table 1). There was no significant difference in peak
PEEP and arterial lactic acid values between the two groups (table 1). Approximately $30 \%$ of patients in both groups had an unstable hemodynamic status necessitating inotropes or vasopressors before intubation (table 1). Anti-viral, anti-microbial, and anti-fungal agents, corticosteroids, and intravenous immunoglobulin were administered at similar frequencies in both groups (table 1). Notably, more patients in the weaned group (44.4\%) than in the nonweaned group $(11.5 \%)$ received convalescent plasma (table 1).

\subsection{Descriptive Data Related to Use of ECMO}

Of the 88 enrolled patients, 79 received V-V ECMO therapy (table 2). Of the 7 patients who received veno-arterial (V-A) ECMO, 4 were weaned and 3 failed to wean (table 2). The two patients who received veno-arterio-venous ( $\mathrm{V}-\mathrm{A}-\mathrm{V})$ ECMO failed to wean from ECMO (table 2). One patient in each group underwent a switch of the ECMO mode (table 2). One patient in the weaned group received ECMO therapy while awake and without mechanical ventilation (table 2). Three patients in the weaned group and two in the non-weaned group had undergone repeated ECMO (table 2). Five patients in the weaned group and 7 patients in the non-weaned group experienced interhospital transportation with ECMO run (table 2). The blood flow velocities of ECMO were equivalent in the two groups (table 2).

During the ICU stay, patients in the weaned 
Table 2 ECMO-related parameters and therapies, and outcomes of patients with COVID-19 and supported with ECMO

\begin{tabular}{|c|c|c|c|c|}
\hline Parameters & All patients $(n=88)$ & Weaned $(n=27)$ & Non-weaned $(n=61)$ & $P$ value \\
\hline \multicolumn{5}{|l|}{ ECMO-related parameters } \\
\hline ECMO mode & & & & 0.194 \\
\hline V-V ECMO & $79 / 88(89.8 \%)$ & $23 / 27(85.2 \%)$ & $56 / 61(91.8 \%)$ & \\
\hline V-A ECMO & $7 / 88(8.0 \%)$ & $4 / 27(14.8 \%)$ & $3 / 61(4.9 \%)$ & \\
\hline V-A-V ECMO & $2 / 88(2.3 \%)$ & $0 / 27(0.0 \%)$ & $2 / 61(3.3 \%)$ & \\
\hline Switch of ECMO mode & $2 / 88(2.3 \%)$ & $1 / 27(3.7 \%)^{*}$ & $1 / 61(1.6 \%)^{\dagger}$ & 0.522 \\
\hline Awake ECMO & $1 / 88(1.1 \%)$ & $1 / 27(3.7 \%)$ & $0 / 61(0.0 \%)$ & 0.674 \\
\hline ECMO recommencement after weaning & $5 / 88(5.7 \%)$ & $3 / 27(11.1 \%)$ & $2 / 61(3.3 \%)$ & 0.335 \\
\hline Interhospital transportation with ECMO run & $12 / 88(13.6 \%)$ & $5 / 27(18.5 \%)$ & $7 / 61(11.5 \%)$ & 0.582 \\
\hline Blood flow of ECMO, L/min & $3.70(3.50-4.00)$ & $3.67(3.42-4.00)$ & $3.70(3.50-4.03)$ & 0.798 \\
\hline \multicolumn{5}{|l|}{ ECMO-related therapies } \\
\hline \multicolumn{5}{|l|}{ Transfusion during ICU stay } \\
\hline Red blood cells, units & $14.00(4.00-26.25)$ & $21.00(10.75-35.25)$ & $12.00(4.00-24.00)$ & 0.012 \\
\hline Plasma, mL & $1200.00(400.00-3162.50)$ & $1550.00(687.50-4550.00)$ & $1050.00(312.50-2797.50)$ & 0.090 \\
\hline Platelet, units & $1.00(0.00-5.00)$ & $0.00(0.00-2.00)$ & $2.00(0.00-5.00)$ & 0.128 \\
\hline CRRT & $57 / 88(64.8 \%)$ & $17 / 27(63.0 \%)$ & $40 / 61(65.6 \%)$ & 0.813 \\
\hline Prone position ventilation during ICU stay & $35 / 88(39.8 \%)$ & $12 / 27(44.4 \%)$ & $23 / 61(37.7 \%)$ & 0.551 \\
\hline Received tracheotomy & $23 / 88(26.1 \%)$ & $15 / 27(55.6 \%)$ & $8 / 61(13.1 \%)$ & $<0.001$ \\
\hline \multicolumn{5}{|l|}{ Outcomes } \\
\hline Weaning of mechanical ventilation & $15 / 87(17.2 \%)$ & $15 / 26(57.7 \%)$ & $0 / 61(0.0 \%)$ & $<0.001$ \\
\hline Survival to hospital discharge & $15 / 88(17.0 \%)$ & $15 / 27(55.6 \%)$ & $0 / 61(0.0 \%)$ & $<0.001$ \\
\hline In-hospital death & $65 / 88(73.9 \%)$ & $4 / 27(14.8 \%)$ & $61 / 61(100 \%)$ & $<0.001$ \\
\hline Under hospitalization & $8 / 88(9.1 \%)$ & $8 / 27(29.6 \%)$ & $0 / 61(0.0 \%)$ & $<0.001$ \\
\hline \multicolumn{5}{|l|}{ Durations } \\
\hline Symptom onset to intubation, days & $20.00(15.00-26.00)$ & $20.00(15.00-28.75)$ & $19.00(15.00-26.00)$ & 0.714 \\
\hline Symptom onset to ECMO initiation, days & $23.00(17.00-31.50)$ & $22.00(17.50-27.50)$ & $24.00(17.00-33.00)$ & 0.402 \\
\hline Intubation to ECMO initiation, days & $3.00(1.00-7.00)$ & $2.00(1.00-6.00)$ & $4.00(2.00-8.00)$ & 0.108 \\
\hline Intubation to tracheotomy, days & $25.00(15.50-30.50)$ & $25.00(17.50-32.00)$ & $19.00(14.75-26.75)$ & $0.194^{\S}$ \\
\hline ECMO initiation to death, days & $13.00(4.00-24.00)$ & $67.50(53.50-80.75)$ & $12.00(3.00-21.00)$ & 0.007 \\
\hline Cumulative ECMO support, days & $13.00(5.75-24.25)$ & $14.00(7.50-31.00)$ & $12.00(3.00-21.00)$ & 0.049 \\
\hline Cumulative IMV support, days & $22.50(10.25-36.00)$ & $37.00(17.50-65.00)$ & $19.00(8.75-29.25)$ & $<0.001$ \\
\hline \multicolumn{5}{|l|}{ Length of stay } \\
\hline ICU stay, days & $25.00(10.75-40.00)$ & $45.00(25.00-70.00)$ & $17.00(9.00-30.00)$ & $<0.001$ \\
\hline Hospital stay, days & $30.50(13.75-50.00)$ & $56.00(41.50-105.50)$ & $21.00(11.00-37.00)$ & $<0.001$ \\
\hline \multicolumn{5}{|l|}{ Complications } \\
\hline \multicolumn{5}{|l|}{ Nosocomial infection } \\
\hline Pulmonary infection & $65 / 88(73.9 \%)$ & $21 / 27(77.8 \%)$ & $44 / 61(72.1 \%)$ & 0.578 \\
\hline Catheter related bloodstream infection & $5 / 88(5.7 \%)$ & $3 / 27(11.1 \%)$ & $2 / 61(3.3 \%)$ & 0.335 \\
\hline \multicolumn{5}{|l|}{ Acute organ injury } \\
\hline Cardiac injury & $72 / 85(84.7 \%)$ & $20 / 26(76.9 \%)$ & $52 / 59(88.1 \%)$ & 0.319 \\
\hline Renal injury & $58 / 86(67.4 \%)$ & $16 / 26(61.5 \%)$ & $42 / 60(70.0 \%)$ & 0.442 \\
\hline Liver injury & $50 / 87(57.5 \%)$ & $11 / 27(40.7 \%)$ & $39 / 60(65.0 \%)$ & 0.034 \\
\hline DIC & $6 / 88(6.8 \%)$ & $2 / 27(7.4 \%)$ & $4 / 61(6.6 \%)$ & 1.000 \\
\hline \multicolumn{5}{|l|}{ Hemorrhage } \\
\hline Pulmonary hemorrhage & $2 / 88(2.3 \%)$ & $0 / 27(0.0 \%)$ & $2 / 61(3.3 \%)$ & 1.000 \\
\hline Gastrointestinal hemorrhage & $19 / 88(21.6 \%)$ & $8 / 27(29.6 \%)$ & $11 / 61(18.0 \%)$ & 0.223 \\
\hline Cannula hemorrhage & $4 / 88(4.5 \%)$ & $2 / 27(7.4 \%)$ & $2 / 61(3.3 \%)$ & 0.762 \\
\hline Intra-cranial hemorrhage & $4 / 88(4.5 \%)$ & $0 / 27(0.0 \%)$ & $4 / 61(6.6 \%)$ & 0.308 \\
\hline Spontaneous epistaxis & $2 / 88(2.3 \%)$ & $0 / 27(0.0 \%)$ & $2 / 61(3.3 \%)$ & 1.000 \\
\hline \multicolumn{5}{|l|}{ Other complications } \\
\hline Thrombosis & $4 / 88(4.5 \%)$ & $3 / 27(11.1 \%)$ & $1 / 61(1.6 \%)$ & 0.158 \\
\hline Pneumothorax & $9 / 88(10.2 \%)$ & $0 / 27(0.0 \%)$ & $9 / 61(14.8 \%)$ & 0.052 \\
\hline Hemothorax & $1 / 88(1.1 \%)$ & $1 / 27(3.7 \%)$ & $0 / 61(0.0 \%)$ & 0.674 \\
\hline Pericardial tamponade & $0 / 88(0.0 \%)$ & $0 / 27(0.0 \%)$ & $0 / 61(0.0 \%)$ & \\
\hline
\end{tabular}


(Continued from the last page)

\begin{tabular}{lcccc}
\hline Parameters & All patients $(n=88)$ & Weaned $(n=27)$ & Non-weaned $(n=61)$ & $P$ value \\
\hline Cause of death & & & & \\
Intractable respiratory failure & $16 / 65(24.6 \%)$ & $3 / 4(75.0 \%)$ & $13 / 61(21.3 \%)$ & 0.069 \\
Heart failure & $22 / 65(33.8 \%)$ & $2 / 4(50.0 \%)$ & $20 / 61(32.8 \%)$ & 0.873 \\
Lethal hemorrhage & $6 / 65(9.2 \%)$ & $1 / 4(25.0 \%)$ & $5 / 61(8.2 \%)$ & 0.816 \\
MODS & $55 / 65(84.6 \%)$ & $4 / 4(100.0 \%)$ & $51 / 61(83.6 \%)$ & 0.869 \\
\hline
\end{tabular}

Data are median (IQR) or $n(\%) . P$ values were calculated by Mann-Whitney $U$ test, $\chi^{2}$ test, or Fisher's exact test, unless specified otherwise. CRRT: continuous renal replacement therapy; IMV: invasive mechanical ventilation; DIC: disseminated intravascular coagulation; MODS: multiple organ dysfunction syndrome. ${ }^{*}$ The patients underwent mode switch from V-V to V-A ECMO. 'The patients underwent mode switch from V-A to V-A-V ECMO. ${ }^{*}$ Awake ECMO indicates that the patient received ECMO therapy while awake and without mechanical ventilation. ${ }^{\S} P$ value was calculated by two-tailed Student's $t$-test.

group received more red blood cell transfusions, with a median of 21.00 units (IQR: $10.75-35.25$; table 2). Plasma and platelet transfusions were similar in both groups (table 2). Up to $64.8 \%$ and $39.8 \%$ of patients received continuous renal replacement therapy and prone position ventilation, respectively, in the ICU. Tracheotomy was performed in $55.6 \%(n=15)$ of patients in the weaned group and $13.1 \%(n=8)$ in the non-weaned group.

\subsection{Outcomes and Complications Associated with Use of ECMO}

Of the 27 patients weaned from ECMO, 15 were further weaned from IMV, 15 were discharged from hospital, 4 died during hospitalization, and 8 remained in hospital (table 2). Of the 61 patients in the nonweaned group, all patients died during hospitalization (table 2). The median time to intubation and ECMO initiation after the onset of symptoms was 20.00 and 23.00 days, respectively, and there was no significant difference between the two groups (table 2). The median (IQR) duration of IMV support before ECMO initiation was $2.00(1.00-6.00)$ days in the weaned group and $4.00(2.00-8.00)$ days in the non-weaned group (table 2). The median (IQR) time from intubation to tracheotomy was $25.00(15.50-30.50)$ days (table 2). The median (IQR) time from ECMO initiation to death was $67.50(53.50-80.75)$ days in the weaned group, and $12.00(3.00-21.00)$ days in the non-weaned group (table 3). The cumulative ECMO support, IMV support, ICU stay, and hospital stay durations were significantly shorter in the non-weaned group than in the weaned group (table 2), which was largely ascribed to the higher mortality rate in the former group.

Microbial culture-confirmed nosocomial pulmonary infection was evident in $73.9 \%$ of patients, and this rate was similar between the two groups (table 2). Cardiac injury was the most common type of acute organ injury, followed by renal and liver injury (table 2). Disseminated intravascular coagulation was observed in $6.8 \%$ of all patients (table 2). Gastrointestinal bleeding was the major hemorrhagic complication (table 2). Pneumothorax was observed exclusively in the non-weaned group (table 2). The major causes of death were multiple organ dysfunction syndrome and heart failure (table 2).

\subsection{Factors Associated with Odds of Successful Weaning from V-V ECMO}

After excluding 9 patients who received V-A ECMO or V-A-V ECMO support (fig. 1), a logistic regression analysis was performed to identify factors associated with the likelihood of successful weaning from V-V ECMO. Factors identified as statistically significant $(P<0.1)$ in a univariate analysis were entered into the multivariate logistic regression analysis. Notably, a lymphocyte count $\leq 0.5 \times 10^{9} / \mathrm{L}$, D-dimer concentration $>4 \times$ ULN at ICU admission and a peak $\mathrm{PaCO}_{2}>60 \mathrm{mmHg}$ at $24 \mathrm{~h}$ before ECMO initiation were independently correlated with lower odds of successful weaning (table 3). A tracheotomy during the ICU stay was independently correlated with higher odds of ECMO weaning (table 3). In contrast, convalescent plasma infusion was significant in the univariate analysis but was not independently associated with the odds of weaning (table 3 ).

\subsection{Comparison of Combined ECMO Therapy and IMV-only Therapy Using Propensity Score- matched Analysis}

Recent EOLIA study questioned the necessity for ECMO to rescue ARDS ${ }^{[1]}$. We then evaluated the effectiveness of ECMO support (ECMO group) and IMV-only support (IMV-only group) for the treatment of COVID-19-related ARDS. To avoid confounding variables that might affect the outcomes of critically ill patients, we performed a propensity score-matched analysis (fig. 1). Moribund patients who died within 24 $\mathrm{h}$ after ECMO or IMV initiation were excluded (fig. 1). We matched 70 patients from the IMV-only group with 70 patients from the ECMO group at a ratio of $1: 1$ in the propensity score-matched analysis (table 4). The crude 120-day in-hospital mortality rates after ICU admission were $74.3 \%$ in the ECMO group and $80.0 \%$ in the IMV-only group (table 4). After applying an adjusted mixed-effect Cox model with the hospital site as a random effect and adjusting the imbalanced 
Table 3 Univariate and multivariate logistic regression analysis of variables between weaned and non-weaned groups

\begin{tabular}{|c|c|c|c|c|c|c|}
\hline \multirow{2}{*}{ Parameters } & \multirow{2}{*}{$\begin{array}{l}\text { Weaned } \\
(n=23)\end{array}$} & \multirow{2}{*}{$\begin{array}{c}\text { Non-weaned } \\
(n=56)\end{array}$} & \multicolumn{2}{|c|}{ Univariate logistic analysis } & \multicolumn{2}{|c|}{ Multivariate logistic analysis } \\
\hline & & & OR $(95 \% \mathrm{CI})$ & $P$ value $^{*}$ & OR $(95 \% \mathrm{CI})$ & $P$ value \\
\hline \multicolumn{7}{|c|}{ Clinical characteristics on hospital admission } \\
\hline Age $\geq 60$, years & $8 / 23(34.8 \%)$ & $33 / 56(58.9 \%)$ & $0.372(0.135-1.020)$ & 0.055 & $0.095(0.008-1.174)$ & 0.067 \\
\hline Female & $8 / 23(34.8 \%)$ & $23 / 56(41.1 \%)$ & $0.765(0.279-2.101)$ & 0.603 & & \\
\hline Major comorbidities & $11 / 23(47.8 \%)$ & $28 / 56(50.0 \%)$ & $0.917(0.347-2.422)$ & 0.861 & & \\
\hline Hypertension & $7 / 23(30.4 \%)$ & $23 / 56(41.1 \%)$ & $0.628(0.223-1.768)$ & 0.378 & & \\
\hline Diabetes mellitus & $6 / 23(26.1 \%)$ & $10 / 56(17.9 \%)$ & $1.624(0.512-5.153)$ & 0.411 & & \\
\hline \multicolumn{7}{|c|}{ Laboratory examinations on ICU admission } \\
\hline Lymphocyte count $\leq 0.5 \times 10^{9} / \mathrm{L}$ & $5 / 23(21.7 \%)$ & $26 / 56(46.4 \%)$ & $0.321(0.104-0.984)$ & 0.047 & $0.021(0.001-0.756)$ & 0.035 \\
\hline Platelet $<$ LLN,$\times 10^{9} / \mathrm{L}$ & $5 / 23(21.7 \%)$ & $20 / 56(35.7 \%)$ & $0.500(0.161-1.550)$ & 0.230 & & \\
\hline C-reactive protein $\geq 100 \mathrm{mg} / \mathrm{L}$ & $11 / 22(50.0 \%)$ & $24 / 55(43.6 \%)$ & $1.292(0.479-3.480)$ & 0.613 & & \\
\hline Total bilirubin $>\mathrm{ULN}, \mu \mathrm{mol} / \mathrm{L}$ & $3 / 23(13.0 \%)$ & $17 / 56(30.4 \%)$ & $0.344(0.090-1.315)$ & 0.119 & & \\
\hline $\mathrm{LDH}>1.5 \times \mathrm{ULN}, \mathrm{U} / \mathrm{L}$ & $18 / 20(90.0 \%)$ & $43 / 51(84.3 \%)$ & $1.674(0.323-8.669)$ & 0.539 & & \\
\hline NT-proBNP or BNP $>U L N, p g / m L$ & $11 / 23(47.8 \%)$ & $23 / 55(41.8 \%)$ & $1.275(0.480-3.391)$ & 0.626 & & \\
\hline Creatinine $>\mathrm{ULN}, \mu \mathrm{mol} / \mathrm{L}$ & $5 / 23(21.7 \%)$ & $16 / 56(28.6 \%)$ & $0.694(0.220-2.189)$ & 0.534 & & \\
\hline $\mathrm{D}$-dimer $>4 \times \mathrm{ULN}, \mathrm{mg} / \mathrm{L}$ & $12 / 23(52.2 \%)$ & $42 / 55(76.4 \%)$ & $0.338(0.121-0.944)$ & 0.038 & $0.020(0.001-0.405)$ & 0.011 \\
\hline Serum IL- $6 \geq 10 \times U L N, p g / m L$ & $9 / 20(45.0 \%)$ & $17 / 42(40.5 \%)$ & $1.203(0.411-3.525)$ & 0.736 & & \\
\hline \multicolumn{7}{|l|}{ Scores on ICU admission } \\
\hline Murray score $\geq 3$ & $16 / 23(69.6 \%)$ & $42 / 56(75.0 \%)$ & $0.762(0.260-2.231)$ & 0.620 & & \\
\hline SOFA score $\geq 8$ & $12 / 20(60.0 \%)$ & $26 / 48(54.2 \%)$ & $1.269(0.440-3.662)$ & 0.659 & & \\
\hline \multicolumn{7}{|l|}{ 24-h before ECMO initiation } \\
\hline Highest $\mathrm{PaO}_{2} / \mathrm{FiO}_{2}<80, \mathrm{mmHg}$ & $4 / 15(26.7 \%)$ & $17 / 39(43.6 \%)$ & $0.471(0.127-1.740)$ & 0.259 & & \\
\hline Highest $\mathrm{PaCO}_{2}>60, \mathrm{mmHg}$ & $2 / 15(13.3 \%)$ & $25 / 44(56.8 \%)$ & $0.117(0.024-0.581)$ & 0.009 & $0.015(0.001-0.368)$ & 0.010 \\
\hline Lowest arterial $\mathrm{pH}<7.30$ & $3 / 15(20.0 \%)$ & $15 / 44(34.1 \%)$ & $0.483(0.118-1.980)$ & 0.312 & & \\
\hline $\begin{array}{l}\text { Highest arterial lactic acid }>\mathrm{ULN} \text {, } \\
\mathrm{mmol} / \mathrm{L}\end{array}$ & $9 / 15(60.0 \%)$ & $18 / 33(54.5 \%)$ & $1.250(0.362-4.318)$ & 0.724 & & \\
\hline \multicolumn{7}{|l|}{ Treatments during ICU stay } \\
\hline $\begin{array}{l}\text { Requirement of inotropes or } \\
\text { vasopressors before IMV }\end{array}$ & $7 / 23(30.4 \%)$ & $15 / 56(26.8 \%)$ & $1.196(0.411-3.476)$ & 0.743 & & \\
\hline $\begin{array}{l}\text { Prone position ventilation during } \\
\text { ICU stay }\end{array}$ & $11 / 23(47.8 \%)$ & $20 / 56(35.7 \%)$ & $1.650(0.617-4.414)$ & 0.319 & & \\
\hline Received tracheotomy & $14 / 23(60.9 \%)$ & $8 / 56(14.3 \%)$ & $9.333(3.036-28.695)$ & 0.000 & $29.021(1.458-577.797)$ & 0.027 \\
\hline Convalescent plasma & $10 / 23(43.5 \%)$ & $6 / 56(10.7 \%)$ & $6.410(1.966-20.900)$ & 0.002 & $18.795(0.108-3267.396)$ & 0.265 \\
\hline \multicolumn{7}{|l|}{ Durations } \\
\hline $\begin{array}{l}\text { Intubation to ECMO initiation } \\
\geq 7 \text {, days }\end{array}$ & $4 / 22(18.2 \%)$ & $16 / 56(28.6 \%)$ & $1.800(0.527-6.151)$ & 0.348 & & \\
\hline
\end{tabular}

Data are $n$ (\%). Upper limit of normal (ULN) and lower limit of normal (LLN) were defined according to the normal ranges of tests in each hospital. LDH: lactate dehydrogenase; NT-proBNP: N-terminal pro-brain natriuretic peptide; BNP: brain natriuretic peptide; IL-6: interleukin-6; SOFA: sequential organ failure assessment; $\mathrm{PaO}_{2} / \mathrm{FiO}_{2}$ : partial pressure of arterial oxygen/fraction of inspiration oxygen; $\mathrm{PaCO}_{2}$ : partial pressure of arterial carbon dioxide; $\mathrm{OR}$ : odds ratio; $\mathrm{CI}$ : confidence interval. ${ }^{*} P$ values were calculated based on univariate logistic regression models. ${ }^{\dagger} P$ values were calculated based on multivariate logistic regression models.

parameters, the results demonstrated a significantly lower risk of 120-day in-hospital mortality after ICU admission in the ECMO group (adjusted HR, 0.479; 95\% CI, 0.290-0.792, $P=0.00410)$ as compared with that in the IMV-only group (fig. 3).

\section{DISCUSSION}

Herein, we report the descriptive data collected from 7 ECMO centers in Wuhan during the COVID-19 pandemic, which included almost all of the ECMO cases in Wuhan. To the best of our knowledge, this is the largest cohort of ECMO-treated patients that has been reported since the COVID-19 outbreak. Altogether, 94 patients received ECMO treatment. Among them, 27 patients were successfully weaned from ECMO, 15 were fully recovered and discharged from the hospital, 8 remained in hospital, and 65 died. The crude inhospital mortality rate of patients who received ECMO therapy in Wuhan during the COVID-19 pandemic was $73.9 \%$. This rate is in contrast to a previous study that reported a mortality rate of $32 \%$ during the H1N1 pandemic in $2009^{[2]}$ and another study that reported a mortality rate of $65 \%$ during the Middle East respiratory syndrome pandemic in $2012^{[12]}$. The relatively high mortality rate in this study might be attributable to the following factors. (1) Expert ECMO centers and experienced ECMO specialists were lacking during the 


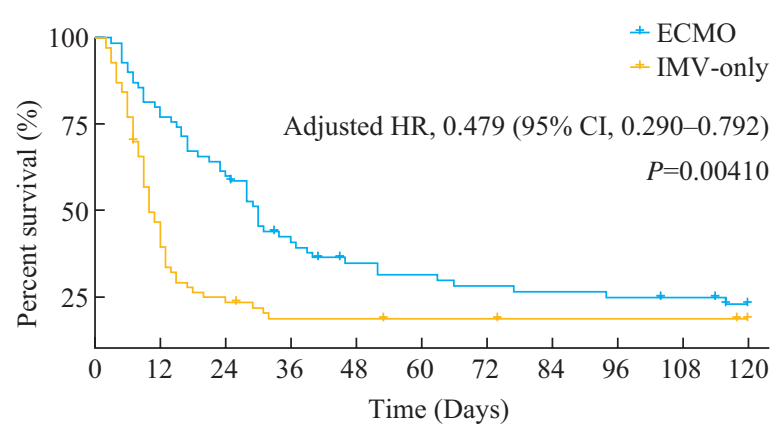

Numbers at risk

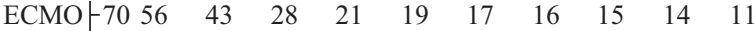

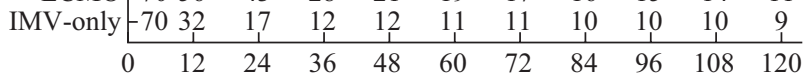
Time (Days)

Fig. 3 Kaplan-Meier curves of the cumulative probability of in-hospital mortality during the 120-day follow-up period after ICU admission (the primary endpoint) in the ECMO cohort and propensity-score matched IMVonly cohort. The blips on the curve indicate the censored cases during the 120-day follow-up period. An adjusted mixed-effect Cox model using the hospital site as a random effect was used to detect the hazard ratio (HR) regarding the primary endpoint. $P$ value was calculated based on mixed-effect Cox model. HR, hazard ratio; CI, confidence interval
COVID-19 outbreak, especially during the early phases when medical systems were overwhelmed. From January 24 to March 8, 2020, multiple medical teams and additional ECMO resources were dispatched from all over the nation to Wuhan to combat COVID-19. On February 2, a stringent quarantine policy was implemented, resulting in a decreasing trend in the daily number of confirmed cases thereafter ${ }^{[13]}$. At that time, the shortages of medical resources began to be alleviated, especially in the ICUs. However, ECMO as a salvage therapy might not have been effective in critically ill patients for whom timely treatment had been delayed, leading to substantial irreversible hypoxemic injuries to various organs. (2) Knowledge regarding the etiology, progression dynamics, and effective therapies was scarce during the early phases of the COVID-19 outbreak. Deaths related to this limited experience were reflected by an ECMO-related mortality rate that approached $92 \%$ in January 2020 . (3) Expertise in ECMO varied among hospitals, which led to significant 3- to 4-fold differences in successful weaning between hospitals (data not shown). Hence, centralized management of multidisciplinary care at high-volume ECMO therapy centers would be ideal.

Table 4 Characteristics of patients receiving ECMO support and IMV-only support after propensity score matching

\begin{tabular}{|c|c|c|c|}
\hline Parameters & $\operatorname{ECMO}(n=70)$ & IMV-only $(n=70)$ & SD value ${ }^{*}$ \\
\hline \multicolumn{4}{|l|}{ Clinical characteristics on hospital admission } \\
\hline Age $\geq 60$, years & $36 / 70(51.4 \%)$ & $43 / 70(61.4 \%)$ & 0.203 \\
\hline Female gender & $28 / 70(40.0 \%)$ & $28 / 70(40.0 \%)$ & $<0.001$ \\
\hline Any comorbidity & $35 / 70(50.0 \%)$ & $36 / 70(51.4 \%)$ & 0.029 \\
\hline \multicolumn{4}{|l|}{ Major comorbidities } \\
\hline Hypertension & $28 / 70(40.0 \%)$ & $23 / 70(32.9 \%)$ & 0.149 \\
\hline Diabetes mellitus & $15 / 70(21.4 \%)$ & $16 / 70(22.9 \%)$ & 0.034 \\
\hline Chronic lung disease & $0 / 70(0.0 \%)$ & $3 / 70(4.3 \%)$ & 0.299 \\
\hline Cardiovascular disease (excluding hypertension) & $7 / 70(10.0 \%)$ & $4 / 70(5.7 \%)$ & 0.160 \\
\hline Neurological disease & $2 / 70(2.9 \%)$ & $1 / 70(1.4 \%)$ & 0.099 \\
\hline Chronic liver disease & $2 / 70(2.9 \%)$ & $2 / 70(2.9 \%)$ & $<0.001$ \\
\hline Chronic renal disease & $0 / 70(0.0 \%)$ & $2 / 70(2.9 \%)$ & 0.243 \\
\hline \multicolumn{4}{|l|}{ Laboratory examinations on ICU admission } \\
\hline Leukocyte count $>10 \times 10^{9} / \mathrm{L}$ & $47 / 70(67.1 \%)$ & $47 / 68(69.1 \%)$ & 0.042 \\
\hline Lymphocyte count $\leq 0.8 \times 10^{9} / \mathrm{L}$ & $47 / 70(67.1 \%)$ & $47 / 68(69.1 \%)$ & 0.042 \\
\hline Platelet $<\mathrm{LLN} \times 10^{9} / \mathrm{L}$ & $22 / 70(31.4 \%)$ & $16 / 70(22.9 \%)$ & 0.194 \\
\hline C-reactive protein $>100 \mathrm{mg} / \mathrm{L}$ & $30 / 69(43.5 \%)$ & $23 / 57(40.4 \%)$ & 0.063 \\
\hline Total bilirubin $>\mathrm{ULN}, \mu \mathrm{mol} / \mathrm{L}$ & $16 / 70(22.9 \%)$ & $19 / 67(28.4 \%)$ & 0.126 \\
\hline ALT > ULN, U/L & $28 / 70(40.0 \%)$ & $24 / 66(36.4 \%)$ & 0.075 \\
\hline $\mathrm{LDH}>1.5 \times \mathrm{UNL}, \mathrm{U} / \mathrm{L}$ & $53 / 63(84.1 \%)$ & $59 / 66(89.4 \%)$ & 0.156 \\
\hline NT-proBNP or BNP $>\mathrm{ULN}, \mathrm{pg} / \mathrm{mL}$ & $29 / 67(43.3 \%)$ & $26 / 60(43.3 \%)$ & 0.001 \\
\hline $\mathrm{cTnI}>\mathrm{ULN}, \mathrm{pg} / \mathrm{mL}$ & $28 / 67(41.8 \%)$ & $15 / 63(23.8 \%)$ & 0.390 \\
\hline Creatinine $>\mathrm{ULN}, \mu \mathrm{mol} / \mathrm{L}$ & $17 / 70(24.3 \%)$ & $13 / 66(19.7 \%)$ & 0.111 \\
\hline D-dimer $>4 \times$ ULN, mg/L & $48 / 69(69.6 \%)$ & $46 / 61(75.4 \%)$ & 0.131 \\
\hline Serum IL- $6 \geq 10 \times \mathrm{ULN}, \mathrm{pg} / \mathrm{mL}$ & $22 / 55(40.0 \%)$ & $22 / 32(68.8 \%)$ & 0.603 \\
\hline $\mathrm{PaO}_{2}<80, \mathrm{mmHg}$ & $27 / 59(45.8 \%)$ & $19 / 55(34.6 \%)$ & 0.230 \\
\hline $\mathrm{PaCO}_{2}, \mathrm{mmHg}$ & & & 0.195 \\
\hline$>50$ & $22 / 59(37.3 \%)$ & $25 / 54(46.3 \%)$ & \\
\hline$<35$ & $10 / 59(17.0 \%)$ & $9 / 54(16.7 \%)$ & \\
\hline
\end{tabular}


(Continued from the last page)

\begin{tabular}{|c|c|c|c|}
\hline Parameters & $\operatorname{ECMO}(n=70)$ & IMV-only $(n=70)$ & SD value* \\
\hline$\overline{\text { Arterial } \mathrm{pH}}$ & & & 0.667 \\
\hline$>7.45$ & $18 / 55(32.7 \%)$ & $13 / 21(61.9 \%)$ & \\
\hline$<7.35$ & $12 / 55(21.8 \%)$ & $4 / 21(19.1 \%)$ & \\
\hline Arterial lactic acid $>\mathrm{ULN}, \mathrm{mmol} / \mathrm{L}$ & $29 / 48(60.4 \%)$ & $25 / 38(65.8 \%)$ & 0.112 \\
\hline \multicolumn{4}{|l|}{ Scores on ICU admission } \\
\hline Murray score & $3.00(2.70-3.30)$ & $3.33(3.00-3.67)$ & 0.432 \\
\hline Missing data & $0 / 70(0.0 \%)$ & $0 / 70(0.0 \%)$ & \\
\hline SOFA score & $8.00(6.00-10.00)$ & $8.00(7.00-9.00)$ & 0.026 \\
\hline Missing data & $9 / 70(12.9 \%)$ & $21 / 70(30.0 \%)$ & \\
\hline \multicolumn{4}{|l|}{ Treatments during ICU stay } \\
\hline CRRT & $48 / 70(68.6 \%)$ & $27 / 70(38.6 \%)$ & 0.631 \\
\hline Prone position ventilation & $28 / 70(40.0 \%)$ & $24 / 70(34.3 \%)$ & 0.118 \\
\hline Tracheotomy & $21 / 70(30.0 \%)$ & $8 / 70(11.4 \%)$ & 0.471 \\
\hline Convalescent plasma & $16 / 70(22.9 \%)$ & $3 / 70(4.3 \%)$ & 0.563 \\
\hline \multicolumn{4}{|l|}{ Outcomes } \\
\hline Weaning of mechanical ventilation & $13 / 70(18.6 \%)$ & $11 / 70(15.7 \%)$ & 0.076 \\
\hline Survival to hospital discharge & $11 / 70(15.7 \%)$ & $10 / 70(14.3 \%)$ & 0.040 \\
\hline 120-day all-cause mortality after ICU admission during hospitalization & $52 / 70(74.3 \%)$ & $56 / 70(80.0 \%)$ & 0.136 \\
\hline Under hospitalization & $7 / 70(10.0 \%)$ & $4 / 70(5.7 \%)$ & 0.160 \\
\hline \multicolumn{4}{|l|}{ Durations } \\
\hline Symptom onset to first admission, days & $14.00(8.25-21.75)$ & $10.00(7.00-14.00)$ & 0.520 \\
\hline Symptom onset to intubation, days & $20.00(15.25-25.75)$ & $17.00(14.00-23.00)$ & 0.418 \\
\hline Intubation to tracheotomy, days & $25.00(16.00-29.00)$ & $13.00(11.00-31.00)$ & 0.342 \\
\hline Cumulative IMV support, days & $25.00(13.25-37.00)$ & $7.50(5.00-12.00)$ & 1.001 \\
\hline \multicolumn{4}{|l|}{ Length of stay } \\
\hline ICU stay, days & $28.00(15.25-44.00)$ & $10.00(7.00-16.50)$ & 0.877 \\
\hline Hospital stay, days & $32.50(16.25-52.25)$ & $17.50(10.00-26.00)$ & 0.775 \\
\hline
\end{tabular}

Data are median (IQR) or $n(\%)$. Upper limit of normal (ULN) and lower limit of normal (LLN) were defined according to the normal ranges of tests in each hospital. ALT: alanine transaminase; LDH: lactate dehydrogenase; NT-proBNP: N-terminal pro-brain natriuretic peptide; BNP: brain natriuretic peptide; cTnI: cardiac troponin I; IL-6: interleukin-6; $\mathrm{PaO}_{2}$ : partial pressure of arterial oxygen; $\mathrm{PaCO}_{2}$ : partial pressure of arterial carbon dioxide; CRRT: continuous renal replacement therapy; IMV: invasive mechanical ventilation. Age, gender, comorbidities (hypertension, diabetes mellitus), and laboratory examinations on ICU admission (lymphocyte count, C-reactive protein, creatinine, total bilirubin, ALT, LDH, NT-proBNP or BNP, and D-dimer) were used for propensity score matching. "Standardized difference (SD) values were calculated to compare the mean of baseline covariate between ECMO and IMV-only groups.

During the later phases of the COVID-19 pandemic in Wuhan, ECMO patients were transferred between hospitals, leading to improvements in survival.

Our study revealed that a pre-ECMO $\mathrm{PaCO}_{2}>60$ $\mathrm{mmHg}$ was an independent risk factor for a failure to wean from ECMO. This result was consistent with previous studies in which hypercapnia was identified as a marker of poor prognosis related to ECMO therapy ${ }^{[14,15]}$. Moreover, the pre-ECMO $\mathrm{PaCO}_{2}$ was included in the Respiratory ECMO Survival Prediction-Score model used to predict the outcome of ECMO therapy ${ }^{[16]}$. As the COVID-19-induced ARDS progresses, disrupted vasoregulation and massive alveolar microthrombi increase the alveolar dead space and cause a high ventilation-perfusion ratio ${ }^{[17]}$. Additionally, sputum arising from proteinaceous and fibrinous exudates, pulmonary hemorrhage, cell debris, and nosocomial infection all contribute to airway obstruction and lobular consolidation ${ }^{[18]}$. Therefore, hypercapnia is an indicator of progression from type 1 to type 2 respiratory failure and a potential signal of ventilation disorders and disease severity. These phenomena suggest that the reversibility of a COVID-19 patients with severe hypercapnia should be evaluated cautiously before ECMO implementation. In contrast, the implementation of ECMO at an early stage before hypercapnia may lead to a better prognosis.

Zhang et al reported that a D-dimer concentration of $4 \times \mathrm{ULN}$ was the cutoff value for predicting the inhospital death of a COVID-19 patient ${ }^{[19]}$. Our results showed that this D-dimer cutoff was independently correlated with decreased odds of weaning from ECMO, consistent with recent findings suggesting that an elevated D-dimer level predicts a poor outcome in COVID-19 patients ${ }^{[19-21]}$. Moreover, Tang et al demonstrated a correlation of prophylactic heparin use with reduced mortality in patients with D-dimer levels exceeding $3.0 \mathrm{mg} / \mathrm{L}^{[21]}$. D-dimer is a rational marker for disease severity and prognosis because (1) an elevated D-dimer level is caused mainly by inflammation 
associated with COVID-19 and subsequent coagulation activity ${ }^{[19]}$, and (2) massive microvascular thrombosis formation in the alveolar capillaries secondary to vasculitis can trigger ARDS during COVID-19 ${ }^{[18,19]}$.

We and other researchers consistently noted that lymphopenia was an indicator of disease severity and a substantial predictor of an unfavorable outcome of COVID-19 ${ }^{[17,20]}$. Although the underlying mechanisms remain to be elucidated, the main reason likely involves the ability of COVID-19 to induce the exhaustion of lymphocytes, particularly $\mathrm{T}$ lymphocytes ${ }^{[22]}$. As lymphocyte repletion is pivotal in the recovery from COVID-19, ECMO-induced lymphopenia and impaired immunity should be carefully evaluated during treatment ${ }^{[23]}$.

Supportive care remains the mainstay of COVID-19 treatment in the absence of proven effective drugs. Most patients on ECMO also received prolonged IMV support, which requires meticulous airway management. In these patients, tracheotomy would reduce the need for sedation, enable oral intake, facilitate the efficient suction of secretions, allow for early rehabilitation, and facilitate weaning from IMV. We demonstrated that patients who received a tracheotomy had independently increased odds of ECMO weaning, even in the presence of greatly delayed therapy (median duration: 25 days post-intubation). Prolonged intubation and associated sedation would inevitably render the patient vulnerable to ventilatorassociated pneumonia, which would further exacerbate the disease. The observed high mortality rate in the present study may be partially ascribed to the delay in tracheotomy. This delay may be attributable to (i) concerns about tracheotomy-provoked aerosolmediated transmission due to a lack of knowledge about the transmission dynamics of SARS-CoV-2 during the early phases of the pandemic and (ii) concerns about uncontrollable bleeding due to heparization. In fact, tracheotomy might be considered before ECMO initiation. Otherwise, the anticoagulation intensity should be reduced, and deep anesthesia would be required at the time of tracheotomy during an ECMO $\operatorname{run}^{[6]}$

Our propensity score-matched analysis revealed that critically ill COVID-19 patients who received ECMO had a better survival outcome than those who received only IMV. Here, ECMO was frequently used as a last resort when IMV therapy failed to provide oxygenation support, and not as a prophylactic therapy. Our findings were consistent with those of the CESAR study $^{[25]}$ and an analysis of the application of ECMO for H1N1-related ARDS ${ }^{[3]}$. IMV strategies such as a higher PEEP, lung recruitment maneuvers, and a prone position might help during the early phase of refractory hypoxemia. However, most patients with COVID19-related ARDS require a high driving pressure to maintain satisfactory oxygenation and overcome a low lung compliance, which increases the risk of ventilatorinduced lung injury. Although ECMO is expertiseand cost-intensive and carries a higher likelihood of complications, this option provides a full support for oxygenation and $\mathrm{CO}_{2}$ removal, enables a "lung rest" ventilatory strategy, and provides time for virus clearance and lung recovery.

Our study has some limitations. First, the retrospective and uncontrolled nature increases the risk of bias in the study. Patients from 7 hospitals were enrolled, and each patient was treated at the discretion of the treating hospital or even the attending physicians, without a consensus or a standard clinical pathway. Second, the respirator parameters (e.g., plateau pressures, tidal volumes, or PEEPs) were not routinely recorded. This limitation prohibited us from evaluating the role of IMV in the outcome of ECMO use. Third, although we included nearly all ECMO patients in Wuhan, the sample size remained relatively small. Therefore, the strength of our findings might be limited.

Our study provides evidence that lymphocytopenia, a higher D-dimer concentration, and pre-ECMO hypercapnia predict a poor outcome of ECMO use in COVID-19 patients. Tracheotomy, even when delayed, could facilitate weaning from ECMO. Compared with IMV-only support, ECMO therapy was associated with a better survival outcome in critically ill patients with COVID-19-induced ARDS.

\section{Conflict of Interest Statement}

All authors declare no conflict of interest relevant to this study.

\section{REFERENCES}

1 Richardson S, Hirsch JS, Narasimhan M, et al. Presenting characteristics, comorbidities, and outcomes among 5700 patients hospitalized with COVID-19 in the New York city area. JAMA, 2020,323(20):20522059

2 Davies A, Jones D, Bailey $\mathrm{M}$, et al. Extracorporeal membrane oxygenation for 2009 influenza A(H1N1) acute respiratory distress syndrome. JAMA, 2009, 302(17):1888-1895

3 Noah MA, Peek GJ, Finney SJ, et al. Referral to an extracorporeal membrane oxygenation center and mortality among patients with severe 2009 influenza A(H1N1). JAMA, 2011,306(15):1659-1668

4 Li X, Guo Z, Li B, et al. Extracorporeal membrane oxygenation for coronavirus disease 2019 in Shanghai, China. ASAIO J, 2020,66(5):475-481

5 Henry BM, Lippi G. Poor survival with extracorporeal membrane oxygenation in acute respiratory distress syndrome (ARDS) due to coronavirus disease 2019 (COVID-19): Pooled analysis of early reports. J CRIT CARE, 2020,58:27-28

6 Lango R, Szkulmowski Z, Maciejewski D, et al. Revised protocol of extracorporeal membrane oxygenation 
(ECMO) therapy in severe ARDS. Recommendations of the veno-venous ECMO expert panel appointed in February 2016 by the national consultant on anesthesiology and intensive care. Anaesthesiol Intensive Ther, 2017,49(2):88-99

7 Kellum JA, Lameire N, Aspelin P, et al. Kidney disease: Improving global outcomes (KDIGO) acute kidney injury work group. KDIGO clinical practice guideline for acute kidney injury. Kidney Int Suppl, 2012,2(1):1138

8 Lei F, Liu YM, Zhou F, et al. Longitudinal association between markers of liver injury and mortality in COVID-19 in China. Hepatology, 2020,72(2):389-398

9 Gando S, Wada H, Thachil J. Differentiating disseminated intravascular coagulation (DIC) with the fibrinolytic phenotype from coagulopathy of trauma and acute coagulopathy of trauma-shock (COT/ACOTS). J Thromb Haemost, 2013,11(5):826-835

10 Waljee AK, Mukherjee A, Singal AG, et al. Comparison of imputation methods for missing laboratory data in medicine. BMJ OPEN, 2013,3(8):e2847

11 Combes A, Hajage D, Capellier G, et al. Extracorporeal membrane oxygenation for severe acute respiratory distress syndrome. New Engl J Med, 2018,378(21):19651975

12 Alshahrani MS, Sindi A, Alshamsi F, et al. Extracorporeal membrane oxygenation for severe Middle East respiratory syndrome coronavirus. Ann Intensive Care, 2018,8(1):3

13 Pan A, Liu L, Wang C, et al. Association of public health interventions with the epidemiology of the COVID-19 outbreak in Wuhan, China. JAMA, 2020,323(19):1-9

14 Brogan TV, Thiagarajan RR, Rycus PT, et al. Extracorporeal membrane oxygenation in adults with severe respiratory failure: a multi-center database. Intens Care Med, 2009,35(12):2105-2114

15 Delmas C, Zapetskaia T, Conil JM, et al. 3-month prognostic impact of severe acute renal failure under veno-venous ECMO support: Importance of time of onset. J Crit Care, 2018,44:63-71

16 Schmidt M, Bailey M, Sheldrake J, et al. Predicting survival after extracorporeal membrane oxygenation for severe acute respiratory failure. The respiratory extracorporeal membrane oxygenation survival prediction (RESP) score. Am J Resp Crit Care, 2014, 189(11):1374-1382

17 Nuckton TJ, Alonso JA, Kallet RH, et al. Pulmonary dead-space fraction as a risk factor for death in the acute respiratory distress syndrome. N Engl J Med, 2002,346(17):1281-1286

18 Menter T, Haslbauer JD, Nienhold R, et al. Post-mortem examination of COVID19 patients reveals diffuse alveolar damage with severe capillary congestion and variegated findings of lungs and other organs suggesting vascular dysfunction. Histopathology, 2020,77(2):198209

19 Zhang L, Yan X, Fan Q, et al. D-dimer levels on admission to predict in-hospital mortality in patients with Covid-19. J Thromb Haemost, 2020,18(6):13241329

20 Zhou F, Yu T, Du R, et al. Clinical course and risk factors for mortality of adult inpatients with COVID-19 in Wuhan, China: a retrospective cohort study. Lancet, 2020,395(10229):1054-1062

21 Tang N, Bai H, Chen X, et al. Anticoagulant treatment is associated with decreased mortality in severe coronavirus disease 2019 patients with coagulopathy. J Thromb Haemost, 2020,18(5):1094-1099

22 Wang D, Hu B, Hu C, et al. Clinical characteristics of 138 hospitalized patients with 2019 novel coronavirusinfected pneumonia in Wuhan, China. JAMA, 2020, 323(11):1061-1069

23 Chen G, Wu D, Guo W, et al. Clinical and immunological features of severe and moderate coronavirus disease 2019. J Clin Invest, 2020,130(5):2620-2629

24 Henry BM. COVID-19, ECMO, and lymphopenia: a word of caution. Lancet Respir Med, 2020,8(4):e24

25 Peek GJ, Mugford M, Tiruvoipati R, et al. Efficacy and economic assessment of conventional ventilatory support versus extracorporeal membrane oxygenation for severe adult respiratory failure (CESAR): a multicentre randomised controlled trial. Lancet, 2009, 374(9698):1351-1363

(Received Dec. 17, 2020; accepted Jan. 20, 2021) 